\title{
Effective field theories for rooted staggered fermions
}

\section{Claude Bernard}

Washington University, St. Louis, MO 63130, USA

E-mail: cb@lump.wust l.edu

\section{Maarten Golterman*}

San Francisco State University, San Francisco, CA 94132, USA

E-mail: maartenestars.sfsu.edu

\section{Yigal Shamir}

School of Physics and Astronomy, Raymond and Beverly Sackler Faculty of Exact Sciences Tel-Aviv University, Ramat Aviv, 69978 Israel

E-mail: shamir@post.tau.ac.il

We extend the construction of the Symanzik effective action to include rooted staggered fermions, starting from a generalization of the renormalization-group approach to rooted staggered fermions. The Symanzik action, together with the usual construction of a partially quenched chiral effective theory from a local, partially quenched, fundamental theory, can then be used to derive the chiral effective theory. The latter reproduces rooted staggered chiral perturbation theory.

The XXV International Symposium on Lattice Field Theory

July 30-4 August 2007

Regensburg, Germany

\footnotetext{
${ }^{*}$ Speaker.
} 


\section{Introduction}

When the lattice spacing $a$ of some discretization of QCD is small enough (i.e., $a \Lambda_{Q C D} \ll 1$ ), effective field theories (EFTs) such as the Symanzik effective theory (SET) [1] and chiral perturbation theory (ChPT) [2] can be used to account for lattice artifacts through a systematic expansion in $a \Lambda_{Q C D}$. A key assumption for this to work is that the underlying lattice theory is local.

This raises the question whether the construction of a SET and ChPT can be extended to QCD with staggered fermions if the "fourth-root trick" is used. This trick amounts to taking the fourthroot of the staggered determinant for each physical flavor (up, down and strange). Each staggered quark yields a four-fold degeneracy in the continuum limit (there are four "tastes" for each physical flavor), and taking the fourth root aims at removing this degeneracy. ${ }^{1}$ However, at non-zero lattice spacing this formulation of lattice QCD does not correspond to a local theory [6], and thus the question whether the construction of EFTs can be extended to rooted staggered QCD is raised.

Here we will argue that this can indeed be done, starting from the renormalization-group (RG) analysis of rooted staggered fermions [7]. The intuitive idea is to start with $n_{r}$ replicas of each staggered flavor, leading to the presence of the $n_{r}$-th power of the staggered determinant for that flavor in the lattice path integral. This corresponds to a local theory as long as $n_{r}$ is a positive integer, and the assumption is that EFTs such as the SET and ChPT can be constructed for such $n_{r}$. One then reaches the EFT for the rooted theory by continuing $n_{r} \rightarrow 1 / 4$, which at the lattice QCD level precisely corresponds to taking the fourth root. The problem is, of course, whether this continuation can be carried out, and whether it is unique. At the EFT level, $n_{r}$ dependence arises in two ways: explicitly, through the loops calculated in the EFT, but also implicitly, through the dependence of the coefficients of the EFT (the Symanzik coefficients in the SET, and the lowenergy constants (LECs) in the chiral theory) on $n_{r}$. We will argue that the continuation exists, and is unique, if one works to a fixed order in the lattice spacing and in the number of loops in the EFT. At the ChPT level, we will show that the proper EFT is staggered ChPT (SChPT), with the replica rule reproducing the effects of rooting. In other words it is rooted staggered ChPT (rSChPT) [8].

We note that a complementary argument for the validity of rSChPT already exists [9]. A key difference with the current work is that Ref. [9] argues completely within the context of chiral effective theories, starting from a case (four degenerate flavors) where the chiral theory is known because the rooting is trivial. Here, we start instead from the fundamental theory on the lattice and show how rSChPT may be derived from it, via the SET. The replica rule is given definite meaning in the fundamental theory, so its appearance in the EFTs is completely natural. In contrast, the replica rule in Ref. [9] has meaning only at the chiral level. Further, Ref. [9] uses certain plausible, but unproven, assumptions on decoupling and on the analyticity of the expansion around positive quark mass. In particular, the decoupling assumption leaves a small potential loophole. While the three-flavor chiral theory goes over, in the continuum limit, to the standard three-flavor chiral theory of QCD, it is not guaranteed that the LECs have the same numerical values as in QCD. The current argument dispenses with several of the assumptions of Ref. [9] and closes the loophole: The continuum low-energy constants are automatically those of QCD with the correct number of flavors. On the other hand the current argument, based as it is on Ref. [7], inherits the assumptions of that work. The key assumptions are two: For any $n_{r}$, integrating out ultra-violet fermionic

\footnotetext{
${ }^{1}$ For a review of rooted staggered fermions relevant for this work, see Ref. [3]. For general reviews, see Refs. [4, 5].
} 
degrees of freedom modifies the effective gauge-field action by local terms. In addition, because (it is highly plausible that) the theory is renormalizable for any $n_{r}$, the perturbative scaling laws apply even though the underlying theory is non-local. (For more discussion of these assumptions, see also Ref. [3].) Both the present arguments and those of Ref. [9] rely heavily on the validity of the standard partially quenched chiral theory [10] for describing partially quenched fundamental theories that are local. We also need to assume here that the SET exists for partially-quenched theories, as long as the lattice theory is local.

The existence of valid EFTs at non-zero lattice spacing is very important in practice. Even at $a=0.06 \mathrm{fm}$, and with the use of improved staggered fermions, lattice artifacts, such as taste splittings in hadronic multiplets, are significant, and one has to use EFT techniques that incorporate lattice artifacts to obtain good fits [11]. Note that the link established here between the validity of rSChPT and the RG analysis of Ref. [7] turns any numerical test of the EFT framework into a more direct test of the RG picture of the continuum limit of QCD with rooted staggered quarks.

In the next section, we review the RG framework. We employ that framework in Sec. 3 to generalize the staggered theory in a way that will turn out to be useful for our goal, and we present our main argument. In Sec. 4 we clarify the way one of the most important staggered symmetries, shift symmetry, works at the EFT level, and we end with our conclusions. This talk gives a brief account of a more detailed article in preparation [12].

\section{The renormalization-group framework}

The RG approach of Ref. [7] starts from the staggered Dirac operator in the "taste basis" [13]:

$$
D_{\text {taste }}^{-1}=\frac{1}{\alpha}+Q D_{\text {stag }}^{-1} Q^{\dagger}
$$

where $Q$ is the gauge-covariant unitary transformation taking the staggered Dirac operator from the one-component to the taste basis. The only new element here is the addition of the contact term $1 / \alpha$. Contact terms like this arise naturally when one defines a series of blocked staggered Dirac operators through gaussian RG blocking, and one may indeed implement Eq. (2.1) as a "zeroth" blocking step in which no thinning out of degrees of freedom occurs [7].

After carrying out $n$ blocking steps, the partition function may be written as

$$
Z\left(n_{r}\right)=\int \mathscr{D} \mathscr{U} \prod_{k=0}^{n} \mathscr{D} \mathscr{V}^{(k)} \mathbf{B}_{n}\left(n_{r} ; \mathscr{U}, \mathscr{V}^{(k)}\right) \operatorname{Det}^{n_{r}}\left(D_{\text {taste }, n}\right)
$$

with $n_{r}$ is the number of replicas. For simplicity we consider the case of mass-degenerate flavors, with a common quark mass $m$. For now we will take $n_{r}$ to be a positive integer. $D_{\text {taste }, n}$ is the staggered Dirac operator after $n$ hypercubic blocking steps, and $\mathscr{V}^{(k)}$ are the blocked gauge fields. As made explicit in Eq. (2.2), we postpone the integration over gauge fields in order to keep the action bilinear in the fermions, or, equivalently, to express the fermionic part of the partition function as a determinant. We will not need the detailed form of $\mathbf{B}_{n}$, but only note that, under the assumptions of Ref. [7], it represents a local Boltzmann weight on the coarse lattice reached after $n$ blocking steps. The coarse lattice spacing $a_{c}$ is related to the original lattice spacing $a_{f}=a$ by $a_{c}=2^{n+1} a_{f}$ (on the taste basis the lattice spacing is $2 a_{f}$ ), and we choose $a_{f}$ and $n$ such that $a_{c} \Lambda_{Q C D} \ll 1$. 
So far, we have been following a standard RG set up. We now define

$$
\tilde{D}_{i n v, n}=\frac{1}{4} \operatorname{tr}_{\text {taste }}\left(D_{\text {taste }, n}\right)
$$

and use this to split $D_{\text {taste, } n}$ into a taste-invariant and a taste-breaking piece:

$$
D_{\text {taste }, n}=\tilde{D}_{i n v, n} \otimes \mathbf{1}+\Delta_{n}
$$

where 1 is the $4 \times 4$ unit matrix in taste space. It can be shown that, because of the contact term $1 / \alpha$ in Eq. (2.1), $\tilde{D}_{i n v, n}$ has no fermion doublers $[6,14]$. We now assume that the taste-breaking part scales as

$$
\left\|a_{c} \Delta_{n}\right\| \sim \frac{a_{f}}{a_{c}}
$$

which expresses the assumption that taste violations scale to zero when $a_{f} \rightarrow 0$ like a dimensionfive irrelevant operator, in the RG language. Note that, with $n_{r}$ integer, the theory is local, and there is no reason to doubt the existence of the continuum limit.

For $n$ large enough, i.e., $\left\|a_{c} \Delta_{n}\right\|$ small enough, we may expand the theory defined by $D_{\text {taste, } n}$ around the theory defined by $D_{i n v, n}=\tilde{D}_{i n v, n} \otimes \mathbf{1}$. If $\Delta_{n}$ scales as assumed, both theories will have the same continuum limit, ${ }^{2}$ i.e., the continuum limits of the theory with $n_{r}$ staggered fermions, and the theory with $n_{s}=4 n_{r}$ taste-singlet fermions with Dirac operator $\tilde{D}_{i n v, n}$ are the same.

The claim of Ref. [7] is that this conclusion should also hold for the case $n_{r}=n_{s} / 4$, with $n_{s}$ any positive integer, not necessarily a multiple of four. Of course, $n_{r}=1 / 4$ corresponds to a theory with one staggered quark with the fourth root of the staggered determinant. The key point is that the scaling of $\Delta_{n}$ should also hold on the ensemble of gauge configurations generated with $n_{r}=n_{s} / 4$. Next, we will use this as a starting point for the construction of EFTs for rooted staggered QCD.

\section{Generalized theory}

We now generalize the theory defined in Eq. (2.2) by replacing (for details, see Ref. [12])

$$
\operatorname{Det}^{n_{r}}\left(D_{t a s t e, n}\right) \rightarrow \operatorname{Det}^{n_{s}}\left(\tilde{D}_{i n v, n}\right) \frac{\operatorname{Det}^{n_{r}}\left(\tilde{D}_{i n v, n} \otimes \mathbf{1}+t \Delta_{n}\right)}{\operatorname{Det}^{n_{r}}\left(\tilde{D}_{i n v, n} \otimes \mathbf{1}\right)}
$$

This is a theory with $n_{r}$ "generalized" staggered fermions (generalized because of the insertion of the interpolation parameter $t$ ), $n_{s}$ taste-singlet fermions, and $4 n_{r}$ taste-singlet ghosts (i.e., quarks with bosonic statistics). For $t=1$ and $n_{s}=4 n_{r}$ the generalized theory reduces to the staggered theory, Eq. (2.2), while for $t=0$ it is a local "reweighted" theory of $n_{s}$ taste-singlet fermions.

According to our basic assumption, that the SET and ChPT exist for any local discretization of QCD, we may thus assume that the SET, and ChPT, can be constructed along the usual lines for any positive integers $n_{r}$ and $n_{s}$, and any $t$. We will in particular assume that the SET still exists for any fixed integer $n_{s}$, not necessarily equal to $4 n_{r}$ (while still keeping $n_{r}$ integer), for any value of $t$. In other words, we will assume that the SET exists for partially-quenched theories [10], as long as the lattice theory is local. Since $\tilde{D}_{i n v, n}$ is defined on the coarse lattice, the theory defined by the

\footnotetext{
${ }^{2}$ The continuum limit is defined by taking $n \rightarrow \infty$, keeping $a_{c}$ fixed.
} 
replacement (3.1) depends on both the fine and coarse lattice spacings $a_{f}$ and $a_{c}$, and we will think about the SET as an expansion in $a_{f}$, with coefficients that depend on $a_{c}$.

The key observation is that the determinant ratio in Eq. (3.1) can be expanded in $t$, if $\Delta_{n}$ is small enough (i.e., if the number of RG steps $n$ is large enough):

$$
\frac{\operatorname{Det}^{n_{r}}\left(\tilde{D}_{i n v, n} \otimes \mathbf{1}+t \Delta_{n}\right)}{\operatorname{Det}^{n_{r}}\left(\tilde{D}_{i n v, n} \otimes \mathbf{1}\right)}=\exp \left[n_{r} \operatorname{Tr} \log \left(1+t\left(\tilde{D}_{i n v, n}^{-1} \otimes \mathbf{1}\right) \Delta_{n}\right)\right] .
$$

Since $\Delta_{n}$ scales like $a_{f}$, and the trace over taste indices of $\Delta_{n}$ vanishes, it is easy to see that this expansion is really a combined expansion in $t, n_{r}$, and $a_{f}$, with the power of $n_{r}$ smaller than the power of $t$, and the power of $t$ smaller than or equal to that of $a_{f}$ in each term. This implies that when we expand any lattice correlation function to a given fixed order in $a_{f}$, any such correlation function is polynomial in $n_{r} .{ }^{3}$ In the SET that reproduces these correlation functions to that same order in $a_{f}$, the $n_{r}$ dependence comes from Symanzik coefficients as well as from loops. To a given order in the loop expansion, the dependence coming from loops is polynomial in $n_{r}$, and we thus conclude that the Symanzik coefficients have to be polynomial in $n_{r}$ as well. Having established that the SET, including its coefficients as well as all of its correlation functions, depends polynomially on $n_{r}$ to any order in $a_{f}$, it follows that the analytic continuation to non-integer $n_{r}$ exists, and is unique. We may now set $n_{r}=n_{s} / 4$ and $t=1$, obtaining the correct SET for QCD with $n_{s}$ (degenerate) rooted staggered fermions. This is our main result.

We now summarize a number of important comments and consequences. For $t \neq 1$ the SET is complicated, with dependence on both lattice spacings, $a_{f}$ and $a_{c}$. However, we are ultimately interested only in correlation functions at $t=1$, and usually with only staggered fermions on the external legs. In that case, all staggered symmetries (in particular shift symmetry and $U(1)_{\varepsilon}$ symmetry) apply, and it follows that the form of the SET is precisely that of Ref. [15], to order $a_{f}^{2}$. (Ref. [15] only dealt with the case $n_{r}=1$, but it follows from the discussion in Ref. [8] that an analogous form can be written down for integer $n_{r} \neq 1$ as well.) If we now set $n_{r}=n_{s} / 4$, the underlying lattice theory is just lattice QCD with $n_{s}$ rooted staggered quarks, and there is only one lattice spacing $a_{f}$. (While correlation functions will still depend on $a_{f}$ and $a_{c}$, physical quantities can only depend on $a_{f}$.)

The $t$-expansion is an expansion in $\tilde{D}_{\text {inv }, n}^{-1} \Delta_{n}$. This is similar to the expansion in $D_{\text {cont }}^{-1}\left(D_{\text {latt }}-\right.$ $\left.D_{\text {cont }}\right) \sim a p$ underlying the usual construction of the SET, with $p$ the typical momentum on the external legs. Here $\Delta_{n} \sim a_{f} p^{2}$ and $\tilde{D}_{i n v, n}^{-1} \sim 1 / p$, where the momentum $p$ is restricted to be smaller than the cutoff $1 / a_{c}$, because the theory defined by $\tilde{D}_{i n v, n}$ lives on the coarse lattice. It follows that the $t$-expansion is an expansion in powers of $a_{f} / a_{c}$. The theory has no $1 / a_{f}$ divergences, consistent with the fact that the continuum limit is the same for all $t$. (At $t=1$ there are no $1 / a_{f}$ divergences because of staggered symmetries [16], while at $t \neq 1$ the theory is $O\left(a_{f}\right)$ away from the $t=1$ theory.)

The transition to the chiral theory works in the same way, leading us to SChPT with the replica rule (rSChPT) [8, 9] as the correct chiral theory for QCD with rooted staggered fermions. As emphasized above, we need to assume that the chiral theory for the local, but partially quenched, theory with integer $n_{r}$ is a standard (graded-symmetry) one [10].

\footnotetext{
${ }^{3}$ For a careful treatment of external legs, as opposed to the loops arising from the determinant, see Ref. [12].
} 


\section{How shift symmetry works at the effective level}

Shift symmetry plays an important role in the construction of any EFT for QCD with staggered fermions, and here we briefly discuss what shift symmetry looks like at the effective level. Shift symmetry is generated by the transformations

$$
S_{\mu} \chi(x)=\zeta_{\mu}(x) \chi(x+\mu), \quad \zeta_{\mu}(x)=(-1)^{x_{\mu+1}+\ldots+x_{4}},
$$

where $\chi$ is the staggered field in the one-component formulation. From this it follows that any representation of the group takes the form $[16,17]$

$$
\begin{aligned}
& S_{\mu} \rightarrow e^{i a_{f} p_{\mu}} \Xi_{\mu}, \quad\left(-\pi / 2<a_{f} p_{\mu}<\pi / 2\right), \\
& \left\{\Xi_{\mu}, \Xi_{v}\right\}=2 \delta_{\mu v} .
\end{aligned}
$$

However, any continuum EFT is invariant under continuum translations, which, for a translation by a displacement $r$, act on continuum fields as

$$
\phi(p) \rightarrow e^{i p \cdot r} \phi(p) .
$$

We may now combine Eqs. (4.2) and (4.3) with $r$ such that $p \cdot r=-a_{f} p_{\mu}$, and conclude that the EFT is invariant under the group $\Gamma_{4}$ generated by the $\Xi_{\mu}$.

\section{Conclusions}

We have shown that the usual construction of a Symanzik effective theory can be extended to lattice QCD with rooted staggered fermions. An important corollary is that SChPT with the replica rule ( $\mathrm{rSChPT}$ ) is the correct chiral theory for this lattice discretization of QCD. These results follow rather straightforwardly from the RG framework of Ref. [7]; thus an important link is established between the RG-based argument that rooted staggered QCD has the correct continuum limit, and the effective theory already in use for fitting results from numerical computations. Turning this around, the success of such numerical fits constitutes a rather direct test of the validity of the rooted theory. This lends strong support to the conjecture that, while the rooted theory is nonlocal at $a \neq 0$, the non-local behavior is a lattice artifact that can be understood and investigated systematically in an expansion in the lattice spacing.

The generalized lattice theory described by Eq. (3.1) provides us with separate handles on the number of physical flavors, $n_{s}$, and the discretization effects of the original $n_{r}$ staggered fields, which become non-local whenever $n_{r}$ is analytically continued to non-integer values. The continuum limit, in which the number of RG-blocking steps $n$ goes to infinity at fixed coarse lattice spacing $a_{c}$, is a local theory (the coarse-lattice action is a "perfect" action) in the correct universality class, that depends on $n_{s}$ but not on $n_{r}$. The lattice theory itself is independent of $n_{r}$ at $t=0$, and turning on the parameter $t$ re-introduces the $n_{r}$ dependence. Correlation functions, calculated to any given order in $a_{f}$ and to any order in the loop expansion in the effective theories (SET or SChPT), are polynomials in $n_{r}$. We may thus analytically continue $n_{r}$ to $n_{s} / 4$ and arrive at rSChPT.

The argument here is complementary to an earlier argument, formulated within the chiral effective theory itself, in support of the validity of rSChPT for rooted staggered QCD [9]. The 
main new element is the direct linkage between the Symanzik effective theory and the underlying discretization of QCD, in its RG-blocked form. Thus SChPT with the replica rule, derived via the Symanzik effective theory, is now directly tied to the underlying rooted theory. It follows that not only does rSChPT give the correct form of the dependence of physical quantities on the quark mass, but also that the continuum low-energy constants are those of QCD with $n_{s}$ flavors.

For $n_{r}=n_{s} / 4$ with $n_{s}$ not a multiple of four, the theory is non-local, and this non-local behavior is reproduced by the EFT. The way this happens is via the application of the replica rule: one calculates a correlation function in the EFT with $n_{r}$ replicas of staggered fermions, setting $n_{r}=n_{s} / 4$ at the end of the calculation. Once $n_{r}$ has been continued to non-integer values, the correlation functions of the $a_{f} \neq 0$ theory cannot be reproduced by any local lagrangian. For an instructive example of how this works in rSChPT, we refer to the discussion of the scalar meson two-point function to one loop in SChPT in Refs. [9, 12].

\section{Acknowledgments}

CB and MG were supported in part by the US Department of Energy. YS was supported by the Israel Science Foundation under grant no. 173/05.

\section{References}

[1] K. Symanzik, Nucl. Phys. B 226, 187 (1983).

[2] See, for instance, O. Bär, Nucl. Phys. Proc. Suppl. 140, 106 (2005) [arXiv:hep-lat/0409123], S. R. Sharpe, arXiv:hep-lat/0607016.

[3] C. Bernard, M. Golterman and Y. Shamir, PoS LAT2006, 205 (2006) [arXiv:hep-lat/0610003].

[4] S. R. Sharpe, PoS LAT2006, 022 (2006) [arXiv:hep-lat/0610094].

[5] A. S. Kronfeld, talk at this conference.

[6] C. Bernard, M. Golterman and Y. Shamir, Phys. Rev. D 73, 114511 (2006) [arXiv:hep-lat/0604017].

[7] Y. Shamir, Phys. Rev. D 75, 054503 (2007) [arXiv:hep-lat/0607007].

[8] C. Aubin and C. Bernard, Phys. Rev. D 68, 034014 (2003) [arXiv:hep-lat/0304014] and 074011 (2003) [arXiv:hep-lat/0306026].

[9] C. Bernard, Phys. Rev. D 73, 114503 (2006) [arXiv:hep-lat/0603011].

[10] C. W. Bernard and M. F. L. Golterman, Phys. Rev. D 49, 486 (1994) [arXiv:hep-lat/9306005].

[11] C. Aubin et al. [MILC], Phys. Rev. D 70, 114501 (2004) [hep-lat/0407028]; C. Bernard et al. [MILC], PoS LAT2006, 163 (2006) [arXiv:hep-lat/0609053] and poster at this conference.

[12] C. Bernard, M. Golterman and Y. Shamir, in preparation.

[13] H. Kluberg-Stern, A. Morel, O. Napoly and B. Petersson, Nucl. Phys. B 220, 447 (1983).

[14] Y. Shamir, Phys. Rev. D 71, 034509 (2005) [arXiv:hep-lat/0412014].

[15] W. J. Lee and S. R. Sharpe, Phys. Rev. D 60, 114503 (1999) [arXiv:hep-lat/9905023].

[16] M. Golterman and J. Smit, Nucl. Phys. B 245, 61 (1984).

[17] M. F. L. Golterman, Nucl. Phys. B 278, 417 (1986). 